\title{
Magnetic Resonance Imaging of Migrating Neuronal Precursors in Normal and Hypoxic-ischemic Neonatal Rat Brains by Intraventricular MPIO Labeling
}

\author{
Jian Yang ${ }^{1,2}$, Jianxing. $\mathrm{Liu}^{3}$, Gang $\mathrm{Niu}^{2}$, Yong $\mathrm{Liu}^{3}$, and $\mathrm{Ed} \mathrm{X.} \mathrm{Wu}{ }^{1}$
}

\begin{abstract}
In this study, 10-day-old normal rats $(n=6)$ and hypoxic-ischemic $(H-I)$ neonatal rats $(n=6)$ were injected with the micronsized iron oxide particles (MPIOs) into the anterior lateral ventricle. 2D and 3D high-spatial resolution MRI were performed with a 7T animal scanner 1 day before the MPIOs injection and hour 3, day 3, day 7 and day 14 after the MPIOs injection. Intraperitoneal injections of 5-bromo-2'deoxyuridine (BrdU) were used to label newly produced cells, and were given thrice daily for 2 days before sacrifice. Immunohistochemistry and Prussian blue staining indicated that iron particles were inside the nestin+ and BrdU+ neural progenitor cells (NPCs), glial-fibrillary-acidic-protein-positive (GFAP+) astrocytes-like progenitor cells, and neuronal-nuclei-positive $(\mathrm{NeuN}+)$ mature neurons. Here we demonstrate that, in normal neonatal rat brain, the migrating pathway of the endogenous NPCs with MPIO is mainly along the rostral migratory stream to the olfactory bulb. In H-I neonatal rat brain, the migration of endogenous NPCs with MPIO is mainly towards the ischemic regions. Therefore, in vivo magnetic cell labeling of endogenous NPCs with MPIO and subsequently non-invasive, serial MRI monitoring should open up a new approach to probe into the mechanism of cell migration in the developmental brain under physiological and pathologic conditions.
\end{abstract}

\section{INTRODUCTION}

Neural progenitor cells (NPCs) with the capacity of self-renewal and multiple linage differentiation have been well documented to reside the subventricular zone (SVZ) in the forebrain throughout mammalian life span $[1,2]$. In normal rat, the NPCs in the SVZ travel a relatively long distance (about $5 \mathrm{~mm}$ in adult mice) $[2,3]$, mainly along the rostral migratory stream (RMS) to the olfactory bulb (OB) where they differentiate into local interneurons [1-3]. In hypoxic-ischemic (H-I) state, the NPC proliferation and differentiation in the $\mathrm{SVZ}$ is triggered by focal brain lesion and NPCs migrate to the ischemic areas [4,5], suggesting that NPCs play a role in brain self-repairing after ischemia in adult and developmental brain $[4,5]$.

Although numerous studies have investigated the neuroblasts migration using fixed brain tissue [1, 2, 4, 5], there is very limited knowledge based on live imaging of NPCs in the RMS and/or in response to brain H-I insult $[3,6]$. Recently, multiphoton microscope imaging [6] and positron emission tomography [7] were used for non-invasive monitoring of cell migration and metabolism in vivo.

1 Laboratory of Biomedical Imaging and Signal Processing, Department of Electrical and Electronic Engineering, The University of Hong Kong, Hong Kong, China.

2 Medical Imaging Center of The First Hospital, The School of Medicine, Xi'an Jiaotong University, Xi'an, Shannxi Province, China.

3 Institute of neurobiology, Xi'an Jiaotong University, Xi'an, Shannxi Province, China.

Correspondence to: Ed X. Wu, PhD at Tel: (852) 2859-7096, Fax: (852)

2559-8738,Email: ewu@eee.hku.hk
However, they are limited to probing within a few centimeters of depth and cannot provide accurate and $3 \mathrm{D}$ information with high spatial resolution.

A major advantage of using MRI to study cell migration is that the migration can be directly mapped onto the anatomy available from MRI, as well as functional activity available from fMRI or neuronal tract tracing $[3,7,8]$. In the most of MRI-based neural cell tracking, cells were labeled with an MRI contrast agent in culture, and then injected into the animal for imaging. The process of in vitro labeling cell is tedious and it is difficult to maintain the stem cells in undifferentiated states in culture. Recently, Shapiro et al [3] demonstrated that the NPCs can be labeled in vivo. By intraventricular injection of micronsized iron oxide particles (MPIOs), MRI can detect the migrating neural precursors carrying MPIOs along the RMS to the OB in normal adult rats [3]. In this study, we investigate the use of this new approach for in vivo magnetic cell labeling and serial MRI tracking of the endogenous NPCs in postnatal developing rat brains under normal and H-I conditions.

\section{MATERIALS AND METHODS}

The experimental protocol for this study was approved by the Institutional Animal Care and Use Committee.

\section{A. Animal preparation}

All Sprague-Dawley neonatal rats were divided into two groups. Group $1 \quad(n=6)$ : 10-day-old normal rats were stereotactically injected with $10 \mu \mathrm{l}\left(1.25 \times 10^{7}\right)$ of $0.9 \mu \mathrm{m}$ diameter MPIOs (Bangs Laboratories, Inc.) into the anterior left lateral ventricle. The chosen position was $1 \mathrm{~mm}$ caudal from bregma, left $1.5 \mathrm{~mm}$, down $2 \mathrm{~mm}$. Group $2(\mathrm{n}=6)$ : 7-day-old H-I rats (12-16 g) were created by unilateral ligation of left common carotid artery plus exposure to hypoxia for 2 hours at ambient temperatures of $37^{\circ} \mathrm{C}[9,10]$. After 3 days post H-I insult, the MPIOs intraventricular injections were performed with the same protocol as Group 1. Intraperitoneal injections of 5-bromo-2'-deoxyuridine (BrdU $50 \mathrm{mg} / \mathrm{kg}$; Sigma, St. Louis, MO) were given thrice daily for 2 days before sacrifice (see Fig.1).

\section{B. MRI scanning}

All rats were performed MRI using a 7T animal MRI scanner (70/16 Bruker BioSpin MRI PharmaScan, Germany) and a $23 \mathrm{~mm}$ mouse brain coil in 1 day before the MPIOs injection and hour 3, day 3, day 7 and day 14 after the MPIOs injection (see Fig.1). 3D gradient echo MRI was acquired with the following image parameters: TR/TE $=50 / 8 \mathrm{~ms}$, flip angle $=10^{\circ}, \mathrm{FOV}=2.5 \times 2.5 \times 1.2 \mathrm{~cm}^{3}$, spatial resolution $=$ $106 \times 106 \times 100 \mathrm{um}^{3}, \mathrm{NEX}=1$, acquisition time $\approx 23 \mathrm{~min} .2 \mathrm{D}$ gradient echo $\mathrm{T} 2 *$-weighted image $(\mathrm{T} 2 * \mathrm{WI})$ was performed in the transverse direction with TR/TE1/TE2/TE3 = $550 / 8 / 14 / 20 \mathrm{~ms}, \mathrm{NEX}=6, \mathrm{FOV}=2.5 \mathrm{~cm}$, slice thickness $=$ 
$0.3 \mathrm{~mm}$, spatial resolution $=98 \times 98 \mathrm{um}^{2}, 20$ slices, acquisition time $\approx 10 \mathrm{~min}$. T2-weighted image (T2WI) was acquired using a Turbo RARE sequence with TR $=6000 \mathrm{~ms}$, TE $=$ $60 / 200 \mathrm{~ms}, \mathrm{NEX}=2$, spatial resolution $=98 \times 98 \mathrm{um}^{2}, 20$ slices, acquisition time $\approx 6 \mathrm{~min}$.

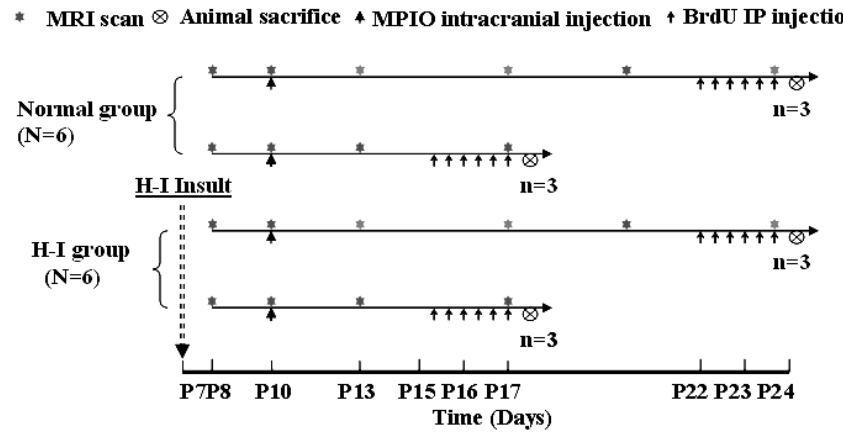

Figure 1. Schedule of H-I insults, MPIO and BrdU IP injections, and MRI scans.

\section{Histology}

After the final MRI scan at different time points (see Fig.1), animals were anesthetized with a dose of ketamine $64 \mathrm{mg} / \mathrm{kg}$ and xylazine $7 \mathrm{mg} / \mathrm{kg}$ intraperitoneally and then perfused transcardially with $10 \%$ formaldehyde. The brains were removed, subsequently fixed and embedded in paraffin. The sagittal and horizontal sections $(8 \mu \mathrm{m})$ were respectively cut in Group 1 and Group 2 rats, and processed for Prussian blue iron staining plus immunohistochemical staining for MPIO in different migrating cells: NPCs (anti-nestin, anti-BrdU), astrocytes-like progenitor cells (anti- GFAP) and mature neurons (anti-NeuN). In Immunocytochemical staining, the sections were treated with $3 \% \mathrm{H}_{2} \mathrm{O}_{2}$ for 20 min followed by antigen repair in $0.01 \mathrm{M}$ Sodium Citrate with microwave, then were treated with $4 \%$ normal goat serum (Boster) for and placed overnight in the primary mouse or rat (for BrdU) antibodies against NeuN (Chemicon, 1:500), GFAP (Neumaker, 1:2000), Nestine (Chemicon, 1:200) and BrdU (BD, 1:1000) respectively. After incubation, sections were washed in $0.01 \mathrm{M}$ PBS and placed for $30 \mathrm{~min}$ in biotinylated anti-mouse (for NeuN, GFAP and Nestine) or anti-rat (for BrdU) IgG secondary antibodies at $37 \mathrm{oC}$. After the section were placed in avidin-biotin complex $(\mathrm{ABC})$ reagent (Boster) for $20 \mathrm{~min}$, they were then reacted in a solution of $0.012 \%$ $\mathrm{H}_{2} \mathrm{O}_{2}$ and $0.05 \%$ 3, 3'-diaminobenzidine (Sigma, St Louis, $\mathrm{MO}, \mathrm{USA}$ ) in TB buffer for $3-5 \mathrm{~min}$, and finally covered.

\section{RESULTS AND DISCUSSION}

A. MRI of migrating neural progenitor cells within the rostral migratory stream in normal noenatal rats

All Group 1 rats were successfully injected with MPIOs in left lateral ventricle. Fig. 2 showed 4 spatially close slices along the left RMS. MRI detected that migration started in day 3 (green arrow), and clearly entered into the RMS pathway in day 7 (blue arrow). In day14 post-injection, MRI exhibited a dark contrast (red arrow) along the RMS and extending into the OB. This migration pattern in 10-day-old neonatal rats is similar to that previously reported in adult rats [3]. However, the dark migrating lines in neonatal rats exhibited more apparent contrast than in adult rats, likely resulting from the enhanced new cell production to meet the developmental requirement [5].

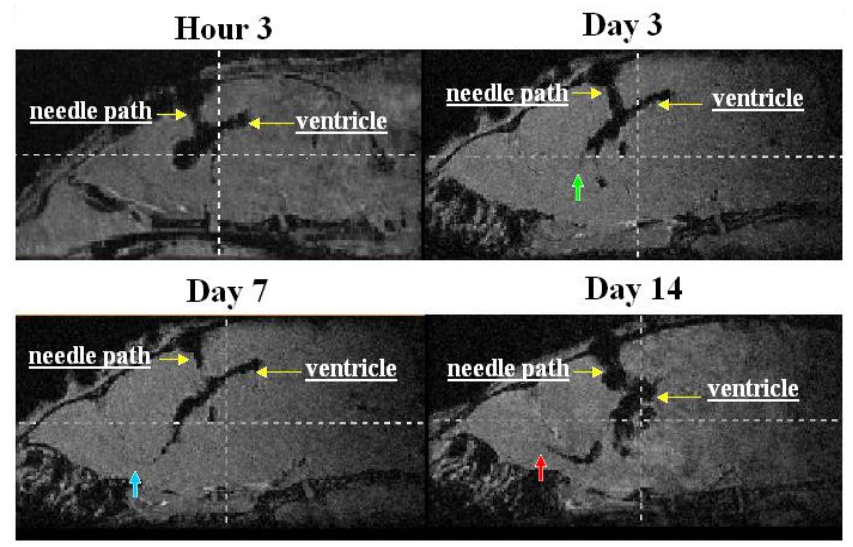

Figure 2. 3D gradient echo MR images in a normal neonatal rat show the migration of endogenous NPCs with MPIOs in a sagittal slice along the left RMS at 4 time points.

B. In vivo MRI detection of migrating neural progenitor cells mainly towards brain lesion in hypoxic-ischemic (H-I) noenatal rats

All Group 2 rats were successfully induced to hypoxicischemic (H-I) models and performed with intraventricular MPIO injections. MRI monitoring of NPCs migration in a severe $\mathrm{H}-\mathrm{I}$ neonatal rat was shown in Fig. 3. At day 14 after MPIO injection, a dramatic migrating territory was present in MRI, that the necrotic regions with high signal intensity were surrounded by irregular dark border. This indicated that the newly produced cells, labeled with MPIOs, migrated to the infarcted regions, likely to replenish the lost neural cells in the region [3].

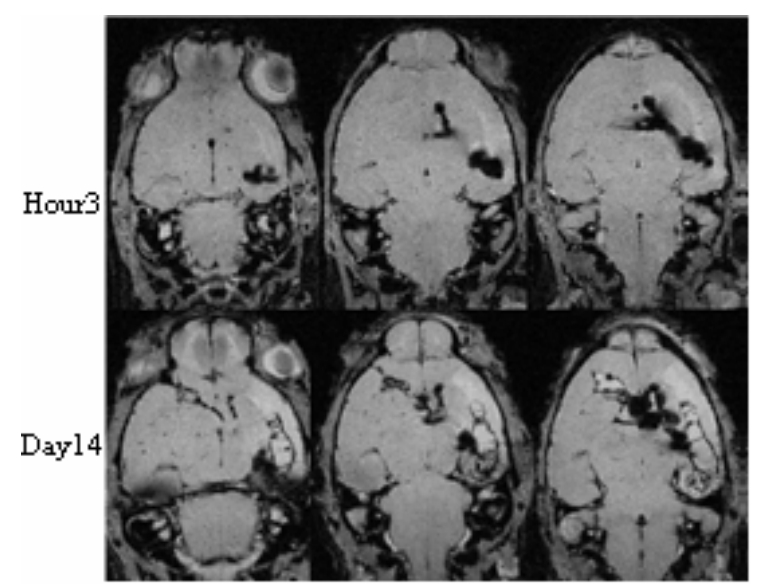

Figure 3. 2D gradient echo MR images (TE=14 ms) in an H-I neonatal rat show the migration of endogenous NPCs with MPIOs in 3 consecutive transverse slices at 2 time points. 


\section{Colocalization of histological findings and MRI}

Histology was performed on the brains to determine what type of cells were labeled by MPIOs along the entire migratory pathway in normal and H-I nenatal rat. The sections stained for iron with Prussian blue and then respectively double stained with nestin and BrdU for NPCs, GFAP for astrocytes-like progenitor cells, and Neu-N for mature neurons. The histological appearances of iron-labeled cells were performed for spatial correlation with MRI findings.

Nestin, an intermediate filament, was first characterized in neuroepithelial stem cells during embryogenesis in rats [11]. In this study, nestin, used as a marker for neural progenitor cells, was expressed in the SVZ and in the migrating neuroblasts in the RMS of normal rat or towards infracted region in $\mathrm{H}-\mathrm{I}$ insult.

In normal neonatal rat, nestin+/iron+ cells were found in the ependymal wall, SVZ, and RMS, and share the features of NPCs and neuroblasts. Moreover, the size of the RMS was wider and the nestin+/iron+ cluster was much darker in neonatal rat than adult rat [6].

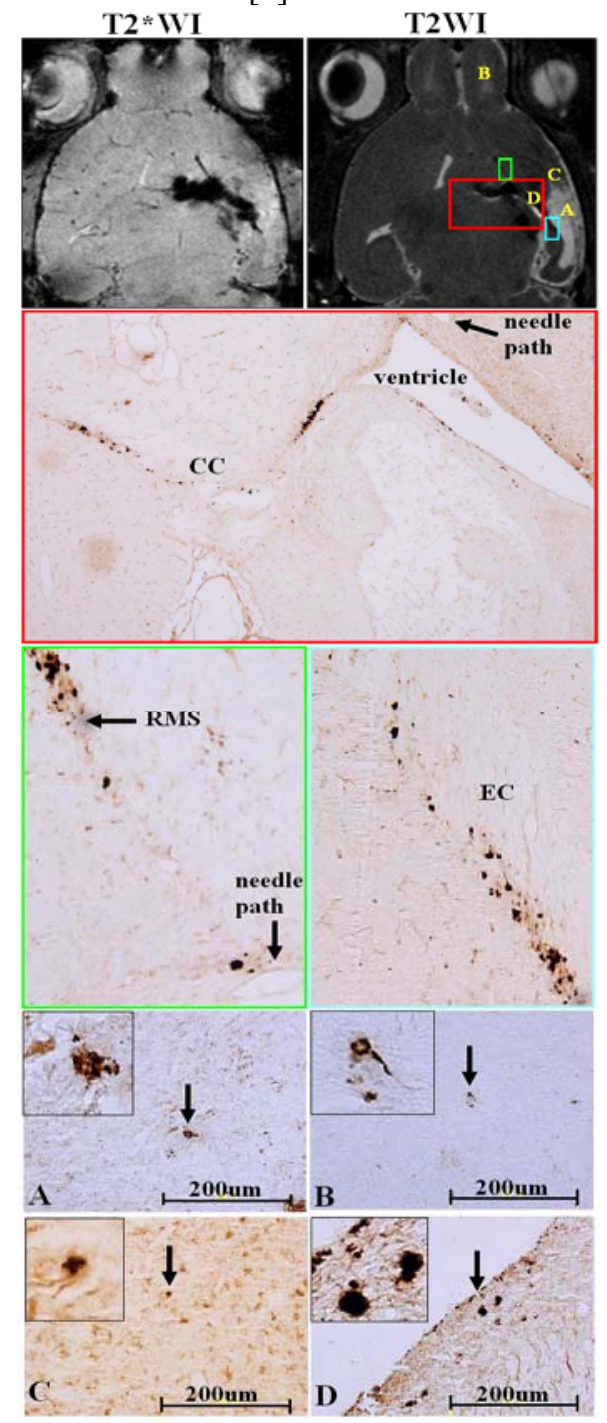

Figure 4. Migration of endogenous NPCs with MPIOs in MRI spatially correlated with the nestin+/iron+ cells in histology in a brain with H-I injury.
As shown in top row of Fig. 4, 2D T2*WI (TE=20ms) and $\mathrm{T} 2 \mathrm{WI}(\mathrm{TE}=60 \mathrm{~ms})$ in a moderate H-I brain (extensive loss of brain tissue with infarction) was acquired in day 7 post-injection, showing the dark lines extending from the needle hole and left ventricle towards RMS and infracted region, and passing through corpus callosum (CC) towards contralateral hemisphere. The $2^{\text {nd }}$ row of Fig. 4 shows the corresponding region marked by the red box in T2WI with a magnified view $(\times 40)$, with the migrating nestin + /iron + cells as dark chains and spots in $\mathrm{CC}$ and the lateral wall of the left ventricle. The images in the $3^{\text {rd }}$ row of Fig. 4 corresponds to the magnified views of the green $(\times 200)$ and blue boxs $(\times 100)$ in T2WI, depicting the migrating nestin+/iron+ cells as the typical chains respectively in RMS towards OB and along the external capsule (EC) towards the infarcted regions. Moreover, the nestin + / iron+ cells were found in the infarcted core (A), OB of ipsilateral side (B), margin of an infarcted region (C) and the lateral wall of the ventricle (D) with a high view $(\times 400)$. These results demonstrated that the endogenous NPCs with MPIOs mainly migrate to infarcted regions and less along RMS to OB, suggesting that NPCs migration route changes in response to the H-I insult, likely as a result of the repairing of the local cell loss. However, the phenomenon that the NPCs with MPIOs migrated to contralateral hemisphere through CC remains to be elucidated.

To establish whether these iron-positive cells are newly generated NPCs, we injected animals with BrdU, which incorporate into cells in S-phase. In H-I rat brains, the majority of these BrdU+/iron+ cells distributed in the lateral wall of the ventricle ( Fig. 5A) and margin of infarcted regions (Fig. 5C). Moreover, the occasional BrdU+/ iron+ cells were found in the infarcted regions (Fig. 5B), RMS and OB of ipsilateral side.

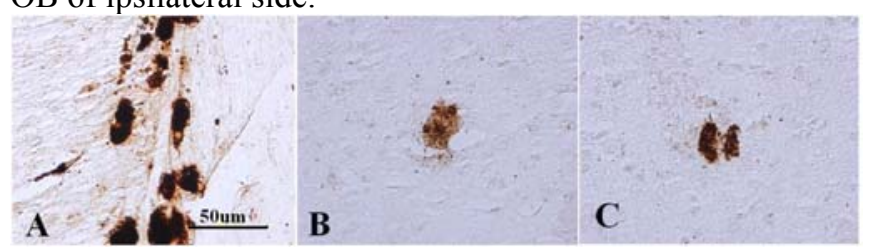

Figure 5. In H-I neonatal rat brains, the migration of endogenous NPCs with MPIOs in the lateral wall of the ventricle (A), the infarcted core (B) and margin of infarcted regions (C) were expressed as the $\mathrm{BrdU}+/$ iron+ cells.

The glial marker GFAP is not only a marker for astrocytes but also a marker of stem/progenitor cells. Again, the guiding astrocytes in the RMS, termed "type B cells", are expressing GFAP [12]. In Fig. 6, a moderate H-I neonatal rat brain was acquired at day 7 post MPIO injection and double-stained with Prussian blue and GFAP. The top image of Fig. 6 shows the astrogliosis in the ipsilateral hemisphere with prominent expression of GFAP at 8 days post $\mathrm{H}-\mathrm{I}$ insult. Corpus callosum (CC) (Fig. 6A), the H-I penumbra (Fig.6B), the lateral wall of the ventricle (Fig.6C) and olfactory bulb of ipsilateral side (Fig.6D) were found with the GFAP+/ iron+ cells. These results demonstrate that the NPCs can differentiate into the astrocytes or/and astrocytes-like progenitor cells with MPIOs and migrate to the remote lesion 
in the form of astrogliosis in H-I injury.

To assess whether the NPCs with MPIOs differentiate into mature neurons, we stained cells with neuronal-nuclei $(\mathrm{NeuN})$. Consistent with their gradual maturation, all of the iron $+/ \mathrm{NeuN}+$ cells had small nuclei (5 10um in diameter), and NeuN was restricted to the nucleus, a phenotype typical of neocortical interneurons [5].

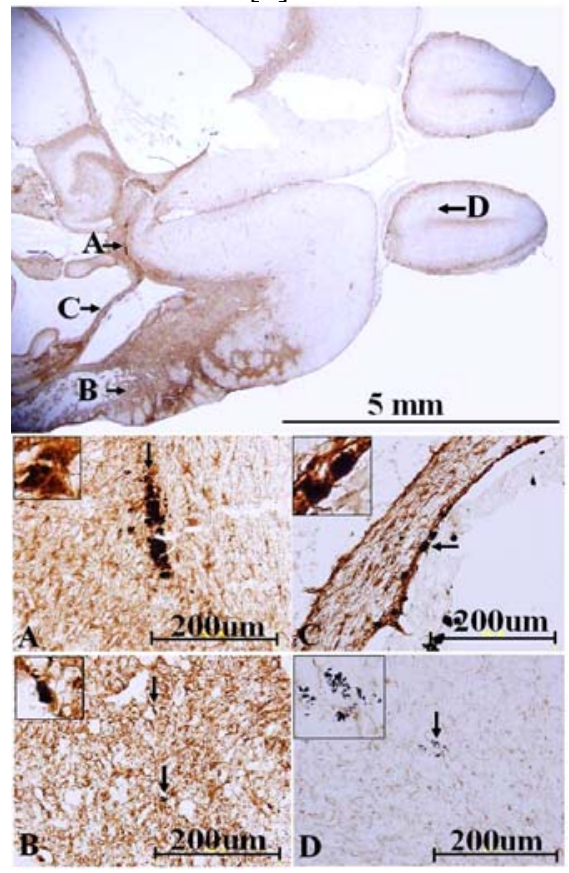

Figure 6. H-I neonatal rat brain double-stained with Prussian blue and GFAP for identifying the migration of astrocytes-like progenitor cells with MPIOs.

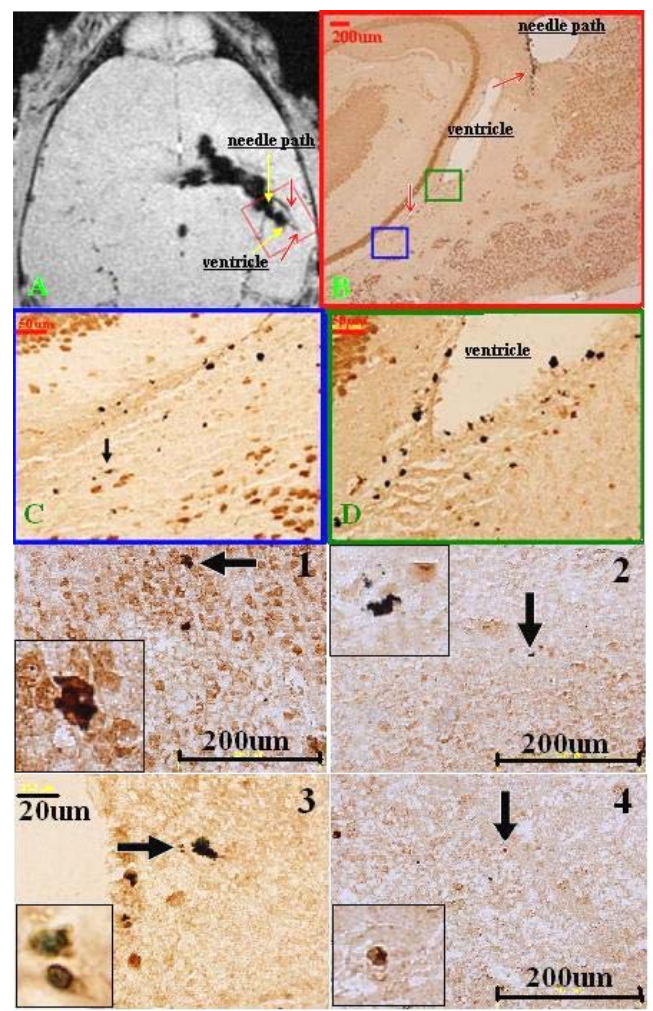

Figure 7. Migration of endogenous NPCs with MPIOs in MRI spatially correlated with the $\mathrm{NeuN}+$ /iron+ mature neurons.
In Fig. 7A, a 2D gradient echo MR image $(\mathrm{TE}=8 \mathrm{~ms})$ in a mild H-I neonatal rat was acquired at day 7 post injection, showing the slight dark lines extend from posterior corner of left ventricle and needle hole (red arrows). The migration of endogenous cells with MPIOs towards the cortical injury boundary was demonstrated by Prussian blue iron staining, and presented as blue-black streams in Fig.7B (red arrow). The Fig.7C and Fig.7D were respectively corresponding to blue and green box with a magnified view. The NPCs with MPIOs, double-stained with iron + and $\mathrm{NeuN}+$, mainly distributed in the H-I penumbra (Fig. 7-1), the lateral wall of the ventricle (Fig. 7-3), and sparsely in the infracted core (Fig. 7-2) and the OB of ipsilateral side (Fig. 7-4), indicating that migrating neuroblasts with MPIOs are capable of differentiating into mature granular interneurons $[3,5]$, and mainly migrate to the injury regions as a self-repairing process after H-I insult in the developmental brains.

\section{CONCLUSION}

In normal neonatal rat brain, the migrating pathway of endogenous NPCs labeled with MPIOs is mainly along the RMS to the OB. In H-I neonatal rat brain, the migration of endogenous NPCs with MPIO is mainly towards the boundary of injured regions. Such migrations can be detected by MRI in vivo within 2 weeks after MPIO injection. In vivo magnetic cell labeling of endogenous NPCs with MPIOs and subsequently non-invasive, serial MRI monitoring should open up a new approach to probe into the mechanisms of cell migration and neuroplasticity in the developmental brains in both normal and pathologic states.

\section{ACKNOWLEDGMENT}

This work was in part supported by Hong Kong Research Grant Council and the University of Hong Kong CRCG grants.

\section{REFERENCES}

[1] F. H. Gage, "Mammalian neural stem cells," Science, vol. 287, pp. 1433-8, Feb 252000.

[2] C. Lois and A. Alvarez-Buylla, "Long-distance neuronal migration in the adult mammalian brain," Science, vol. 264, pp. 1145-8, May 201994.

[3] E. M. Shapiro, O. Gonzalez-Perez, J. Manuel Garcia-Verdugo, A. Alvarez-Buylla, and A. P. Koretsky, "Magnetic resonance imaging of the migration of neuronal precursors generated in the adult rodent brain," Neuroimage, vol. 32, pp. 1150-7, Sep 2006.

[4] Z. Yang and S. W. Levison, "Hypoxia/ischemia expands the regenerative capacity of progenitors in the perinatal subventricular zone," Neuroscience, vol. 139, pp. 555-64, May 122006.

[5] T. Hayashi, M. Iwai, T. Ikeda, G. Jin, K. Deguchi, S. Nagotani, H. Zhang, Y. Sehara, I. Nagano, M. Shoji, T. Ikenoue, and K. Abe, "Neural precursor cells division and migration in neonatal rat brain after ischemic/hypoxic injury," Brain Res, vol. 1038, pp. 41-9, Mar 152005.

[6] L. R. Zhao and S. C. Nam, "Multiphoton microscope imaging: the behavior of neural progenitor cells in the rostral migratory stream," Neurosci Lett, vol. 425, pp. 83-8, Sep 252007.

[7] F. Cicchetti, R. E. Gross, J. W. Bulte, M. Owen, I. Chen, M. Saint-Pierre, X. Wang, M. Yu, and A. L. Brownell, "Dual-modality in vivo monitoring of subventricular zone stem cell migration and metabolism," Contrast Media Mol Imaging, vol. 2, pp. 130-8, May 2007.

[8] R. Guzman, N. Uchida, T. M. Bliss, D. He, K. K. Christopherson, D. Stellwagen, A. Capela, J Greve, R. C. Malenka, M. E. Moseley, T. D. Palmer, and G. K. Steinberg, "Long-term monitoring of transplanted human neural stem cells in developmental and pathological contexts with MRI," Proc Natl Acad Sci U S A, vol. 104, pp. 10211-6, Jun 122007.

[9] Y. Wang, P. T. Cheung, G. X. Shen, I. Bhatia, E. X. Wu, D. Qiu, and P. L. Khong, "Comparing diffusion-weighted and T2-weighted MR imaging for the quantification of infarct size in a neonatal rat hypoxic-ischemic model at 24h post-injury," Int J Dev Neurosci, vol. 25, pp. 1-5, Feb 2007.

[10]J. Yang and E. X. Wu, "Detection of cortical gray matter lesion in the late phase of mild

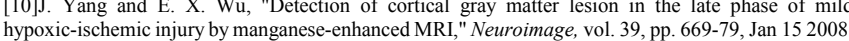

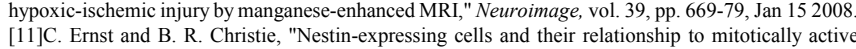
cells in the subventricular zones of the adult rat," Eur J Neurosci, vol. 22, pp. 3059-66, Dec 2005.

[12]M. H. Maurer, R. E. Feldmann, Jr., H. F. Burgers, and W. Kuschinsky, "Protein expression differs between neural progenitor cells from the adult rat brain subventricular zone and olfactory bulb," $B M C$ Neurosci, vol. 9, p. 7, 2008. 